\title{
Transcatheter stent implantation for recurrent pulmonary venous pathway obstruction after the Mustard procedure
}

\author{
Martin C K Hosking, Kenneth A Murdison, Walter J Duncan
}

\begin{abstract}
A 5 year old boy presented with obstruction of the pulmonary venous pathway four years after the Mustard procedure. A successful balloon dilatation of the pulmonary venous pathway was performed but the benefit was transient. Placement of a $10 \mathrm{~mm}$ balloon expandable intravascular stent across the recurrent stenosis resulted in complete relief of the obstruction with prompt resolution of the clinical signs. The delivery system was modified to facilitate stent delivery.
\end{abstract}

(Br Heart f 1994;72:85-88)

Paediatric experience with transcatheter placement of endovascular stents for residual stenosis has expanded from the initial studies of systemic vein and pulmonary artery dilatation to include recent reports of stenting of the ductus arteriosus, aortopulmonary collaterals, and postoperative coarctation of the aorta. ${ }^{12}$ After the Mustard operation for transposition of the great arteries, pulmonary venous pathway obstruction is a less common but more severe complication than systemic venous pathway obstruction. ${ }^{3}$ In an attempt to avoid surgery with its attendant high operative risks balloon dilatation has shown some benefit, although restenosis often occurs. ${ }^{4}$ We report the successful percutaneous intravascular stenting of recurrent pulmonary venous pathway obstruction after a previously successful balloon dilatation. We discuss the technical modifications found useful in facilitating stent placement in locations with difficult access.

\section{Case report}

The 5 year old patient was diagnosed in the immediate newborn period with atrioventricular concordance, ventricular arterial discordance (d-transposition of the great arteries), and intact ventricular septum. A balloon atrial septostomy was performed on the first day of life, then a Mustard procedure at three months.

During the next five years his clinical course was characterised by persistent deterioration in oxygen saturation associated with an increasing requirement for diuretics and supplemental oxygen to maintain adequate oxygenation. Serial precordial Doppler and cross sectional echocardiography showed evidence of progressive pulmonary venous pathway obstruction reaching a maximum flow velocity of $2.0 \mathrm{~m} / \mathrm{s}$ associated with a stenosis measuring $2-3 \mathrm{~mm}$ in diameter. Right ventricular dysfunction was a persistent echocardiographic finding associated with an increase in left ventricle dimension in response to the rise of pulmonary arterial pressure secondary to the pulmonary venous inflow obstruction. A significant systemic to pulmonary venous chamber baffle leak measuring $5 \mathrm{~mm} \times 4 \mathrm{~mm}$ with right to left shunting was seen by Doppler colour flow mapping and contributed to central cyanosis. Serial chest $x$ ray films showed persistence of cardiomegaly with increasing evidence of pulmonary venous congestion.

To avoid the risks of surgical repair in a patient with impaired right (systemic) ventricular function, the initial therapeutic intervention included balloon dilatation of the pulmonary venous pathway obstruction with a $12 \mathrm{~mm} \times 2 \mathrm{~cm}$ balloon (Meditech, Watertown, MA, USA) along with catheter occlusion of the baffle leak with a $17 \mathrm{~mm}$ PDA Rashkind occluder device. ${ }^{5}$ After this, transoesophageal Doppler assessment with simultaneous haemodynamic correlation showed a reduction of the pulmonary venous outlet gradient to $6-8 \mathrm{~mm} \mathrm{Hg}$ with an increase in diameter from $3 \mathrm{~mm}$ to $7 \mathrm{~mm}$. Clinically there was a considerable improvement with reduction in oxygen requirement. Diuretic treatment was no longer required as the pulmonary oedema seen previously cleared.

The benefit was transient and seven months after catheterisation, his clinical state had once again deteriorated with increased oxygen and diuretic requirements as before. Careful imaging and Doppler colour flow mapping again showed a velocity of $2 \mathrm{~m} / \mathrm{s}$ across the pulmonary venous baffle with a recurrence of the stenosis measuring 3-4 mm in diameter. As the previous catheterisation had shown the distensibility of this baffle stenosis, to avoid further restenosis and maintain an optimal passage for pulmonary venous flow we decided to implant an intravascular stent across this area of narrowing.

\section{CATHETERISATION PROCEDURE}

Informed consent was obtained and the procedure was performed under general anaesthesia. A transoesophageal biplane $5 \mathrm{MHz}$ echocardiographic probe was placed before the start of the catheterisation and left in situ throughout. An arterial catheter was placed by a standard percutaneous approach and the 
Figure 1 (A) Angiogram (anterior to posterior projection) of the proximal pulmonary venous atrium showing position and severity of the baffle neck severity of the baffle neck
obstruction (arrows). (B) Anterior to posterior view showing the non-inflated stent (arrows) in position across the baffle obstruction. Note the tortuous catheter course with the guide wire positioned in the left lower pulmonary vein. The delivery sheath has been retracted before inflation. The $17 \mathrm{~mm}$ Rashkind occluder device is in position across a previous systemic to pulmonary venous baffle defect (open arrow) and was used to help position the stent optimally before inflation. (C) Similar view as before showing the inflated stent in position. Note how the stent has attained the curve of the superior baffle aspect (arrows). ruPV, right upper pulmonary vein. upper pulmonary vein;
dPVA, distal pulmonary venous atrium; $p P V A$, proximal pulmonary venous atrium.
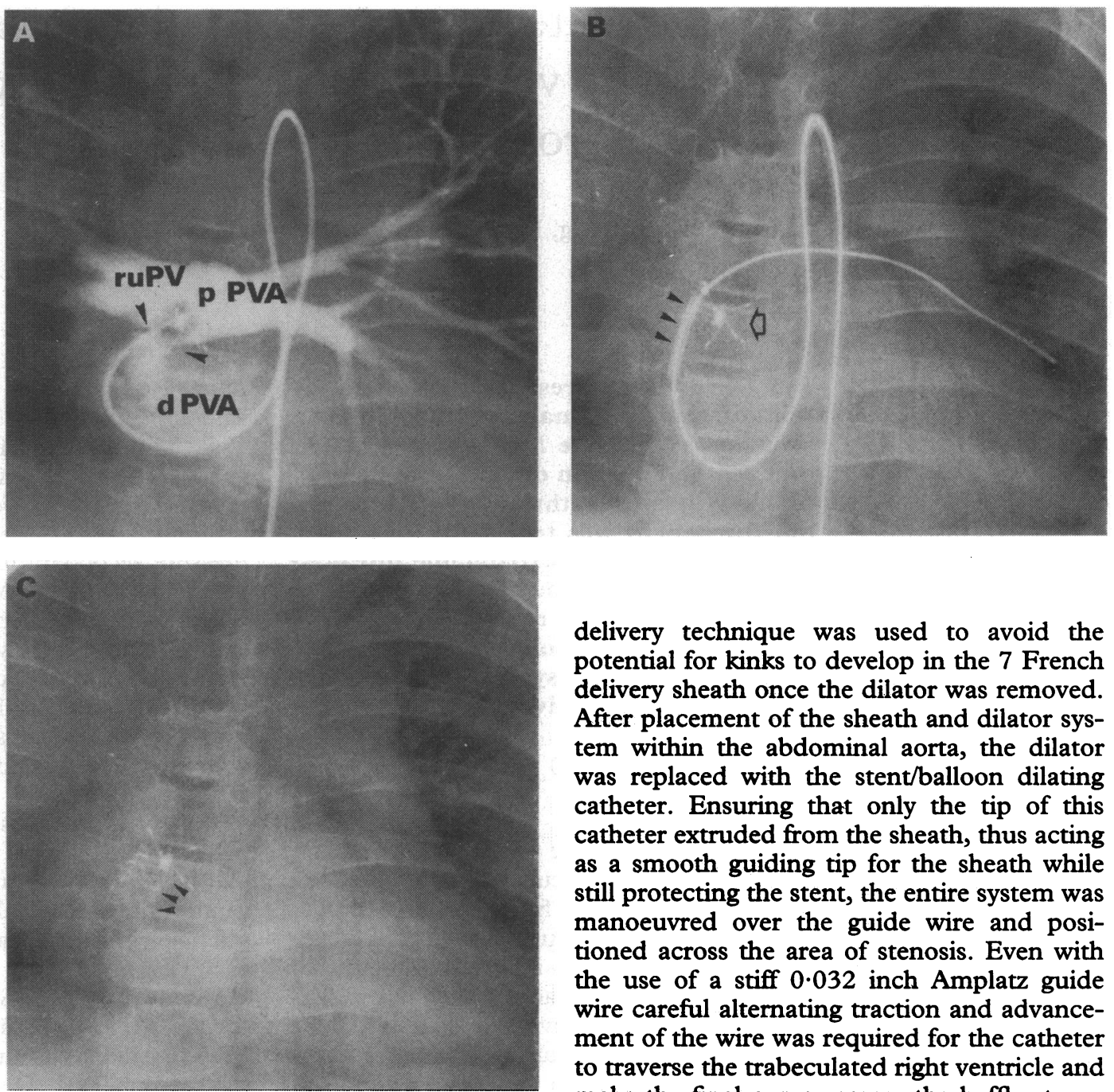

patient was heparinised with $100 \mathrm{U} / \mathrm{kg}$. The diagnosis of pulmonary venous outlet obstruction was confirmed by haemodynamic measurement by pullback tracing over the stenotic area and angiographic assessment (right anterior oblique and lateral projections).

A retrograde arterial approach was used to gain access to the pulmonary venous atrium. An end hole balloon catheter (Arrow International, Reading, PA, USA) was deflected from the morphological right ventricle across the right atrioventricular valve and positioned within the distal pulmonary venous atrium. With the aid of a steerable guide wire (Hi-Torque Floppy, Advanced Cardiovascular systems, Temecula, CA, USA) the area of stenosis was crossed and measured haemodynamically and with angiograms to delineate it (fig 1(A)). A stiff 0.032 inch Amplatz guide wire was positioned in the left lower pulmonary vein and the stent delivery catheter was manoeuvred over this. A $2 \mathrm{~cm}$ Palmaz stent Johnson and Johnson Interventional Systems) carefully secured on a $10 \mathrm{~mm} \times 3 \mathrm{~cm}$ balloon dilating catheter (Optiplast, Mississauga, Ont) was successfully placed across the area of baffle stenosis. The final stent diameter was $10 \mathrm{~mm}$ (fig $1(\mathrm{~A}-\mathrm{C})$ ). Due to the tortuous route leading to the baffle stenosis, a modification in the standard delivery technique was used to avoid the potential for kinks to develop in the 7 French delivery sheath once the dilator was removed. After placement of the sheath and dilator system within the abdominal aorta, the dilator was replaced with the stent/balloon dilating catheter. Ensuring that only the tip of this catheter extruded from the sheath, thus acting as a smooth guiding tip for the sheath while still protecting the stent, the entire system was manoeuvred over the guide wire and positioned across the area of stenosis. Even with the use of a stiff 0.032 inch Amplatz guide wire careful alternating traction and advancement of the wire was required for the catheter to traverse the trabeculated right ventricle and make the final curves across the baffle stenosis. Once in an optimal position, the sheath was withdrawn and the stent deployed in the standard method. No complications occurred during the stenting procedure.

Before stenting, both angiography and transoesophageal echocardiography showed a discrete wedge of tissue projecting into the venous channel from the superior atrial wall resulting in a minimal pulmonary channel of $4 \mathrm{~mm}$ (fig 2A). Haemodynamic measurements showed a mean gradient of $12 \mathrm{~mm} \mathrm{Hg}$, with a maximal Doppler velocity of $1.9 \mathrm{~m} / \mathrm{s}$ and non-laminar colour flow Doppler. After stent placement indwelling transoesophageal echocardiography clearly showed the stent in an optimal position with a widely patent lumen of $10 \mathrm{~mm}$ diameter and laminar unobstructed colour flow Doppler across the open stent (maximal velocity of $0.8 \mathrm{~m} / \mathrm{s}$ ). To avoid potentially dislodging the stent a pressure catheter was not placed across the area and the wire and catheters were removed without complication. The patient was maintained on a heparin infusion $(20 \mathrm{U} / \mathrm{kg} / \mathrm{h})$ for 24 hours then subsequently placed on aspirin (8 $\mathrm{mg} / \mathrm{kg} /$ day).

Since the procedure a dramatic clinical improvement has been seen. His chest $x$ ray film has cleared, there is no further need for diuretic treatment, and oxygen saturations are above $95 \%$ in room air. His activity level and general demeanour have dramatically 


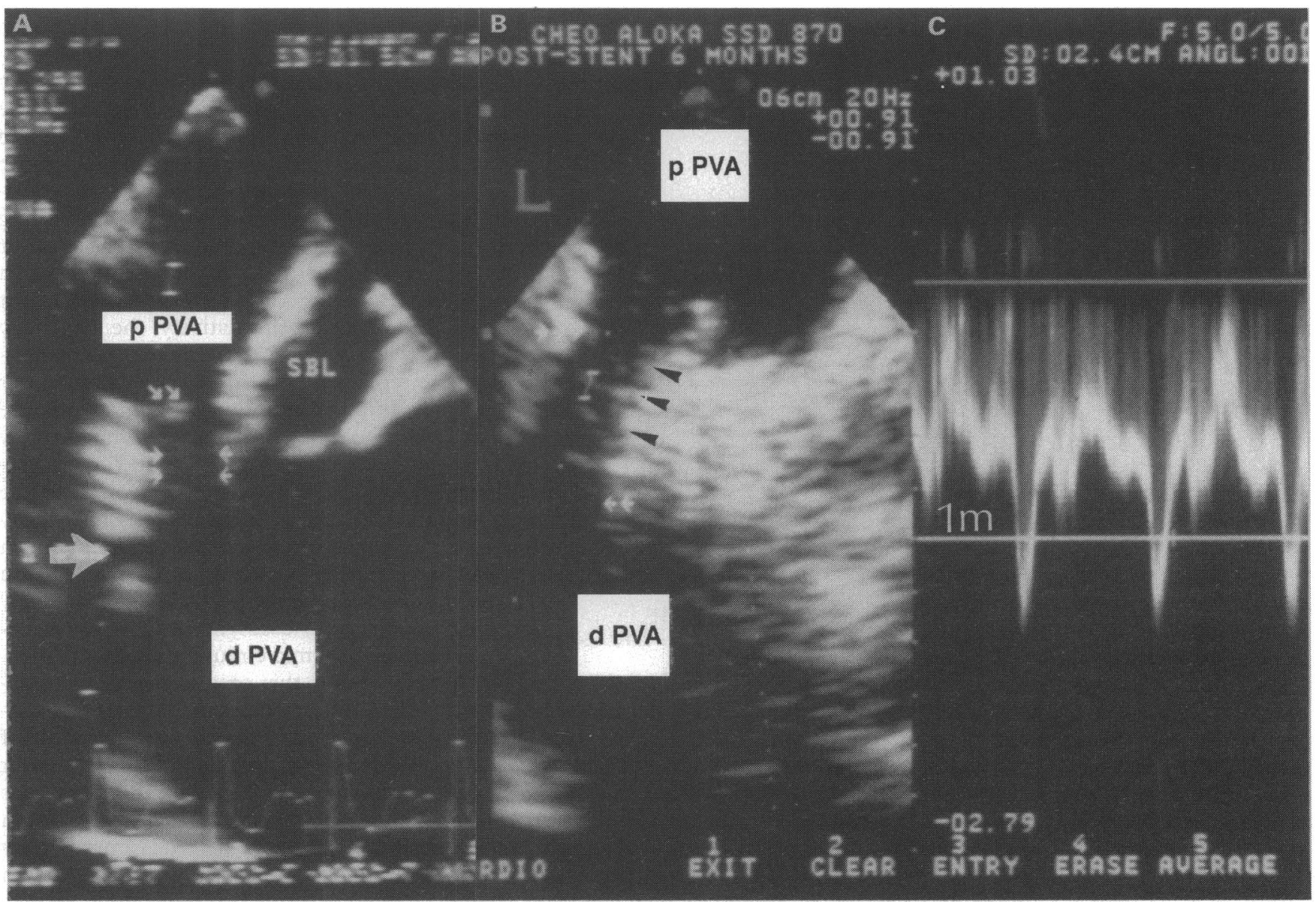

Figure 2 (A) Transoesophageal echocardiogram in the longitudinal plane. There is a discrete 3-4 mm diameter irregular stenosis (arrows) between proximal (p) and distal (d) pulmonary venous atrium (PVA). An additional pulmonary vein (large arrow) is seen to enter the $d P V A$.

(B) Transoesophageal echocardiogram in the longitudinal (L) plane showing the stent in position across the stenosis. Note bow the stent has contact with the superior atrial wall and baffle (arrows) for all of its length, thus providing optimal stability. (C) Doppler spectral analysis of flow after stent implantation.

improved. Follow up Doppler studies have shown persistent relief of the baffle obstruction with flow velocity $<1 \mathrm{~m} / \mathrm{s}$.

\section{Discussion}

In this report we describe the successful application of percutaneous intravascular stenting for the treatment of a persistent pulmonary venous pathway obstruction. Balloon dilatation of a pulmonary venous pathway obstruction has been shown to be an efficacious therapeutic option for this difficult complication of both the Mustard and Senning repair. There is, however, a strong tendency for the stenosis to reoccur ${ }^{4}$ and these patients present a high risk at reoperation. ${ }^{3}$ Intraluminal stents, when inflated, expand with a high resistance to radial collapse thus making them optimal devices for maintaining patency across the distensible venous baffle. One consideration in whether to stent for baffle obstruction is the length of contact between the baffle and the superior atrial wall. This should be sufficient to allow the stent to have adequate atrial wall to baffle contact so as to maintain a stable position when inflated. Care must be made to ensure that, when positioning the stent, the origin of the right upper and right lower pulmonary veins will not be distorted on inflation (fig 1(A)), which could induce a pulmonary vein stenosis. In this case, placement of the stent across the area of restenosis resulted in a widely patent $10 \mathrm{~mm}$ channel with no evidence of stenosis by both imaging and Doppler colour flow mapping. This was associated with a considerable clinical improvement, although close follow up will be required. As the stent is within the systemic circulation we have decided to continue antiplatelet treatment indefinitely, rather than the usual six months for devices placed within the pulmonary circuit or systemic veins.

For pulmonary venous pathway balloon dilation, balloon to stenosis ratios of three to four have been recommended. ${ }^{4}$ As the stent maintains a non-collapsible state after deployment a much smaller balloon to stenosis ratio can be used, thus reducing the potential for arterial damage. A $1 \mathrm{~cm}$ diameter stent was chosen as it was considered that this would allow unimpeded pulmonary venous blood flow. It was recognised that with growth it would be unlikely that a $10 \mathrm{~mm}$ pathway would allow unimpeded flow for life. Further dilatation to 12-14 $\mathrm{mm}$ would have been optimal, but over expansion would result in shortening and distortion of the $2 \mathrm{~cm}$ medium 
Figure 3 Angiogram (lateral projection) during stent inflation showing the anterior position of the ductal occluder device relative to the area of stenosis.

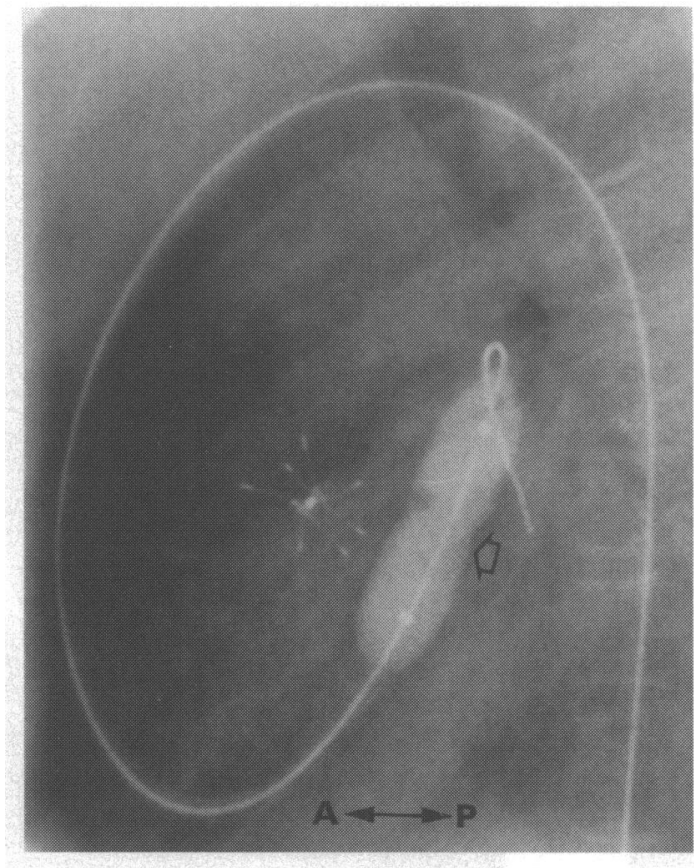

Palmaz stent with the potential for suboptimal positioning on implantation. After complete endothelialisation, the stent will be more secure and not easily displaced. O'Laughlin et al have shown the ability to further dilate implanted stents. ${ }^{1}$ Should flow velocity increase over time an attempt at further inflation to $12 \mathrm{~mm}$ would be a reasonable approach. Close follow up of these patients is mandatory.

Complete endothelialisation of the ductal occluder device occurs within three to six months of implantation. A device that is placed in too posterior a position along the baffle may impinge on systemic and pulmonary venous return. Also it is possible that extension of the endothelialisation process may augment any intrinsic pulmonary venous pathway obstruction. In this case the anterior location of the occluder device relative to the inflated stent (fig 3) makes it unlikely that this would contribute to restenosis.

Due to the tortuous route required to position the stent across the baffle stenosis and as a result of previous experience, we have modified the placement technique from that previously described. In previously described reports after the sheath and dilator have been positioned across the area of stenosis the dilator catheter is removed leaving the guide wire and empty sheath in place. ${ }^{1}$ On removal of the dilator catheter there is a tendency to develop kinks within the sheath especially in those cases where the catheter is needed to negotiate acute angles. As a result, when attempting to pass the balloon dilatation or stent catheter through the sheath it can become caught in the kink, causing displacement of the stent from the balloon. Alternatively, the stent may be unable to traverse the kink thus necessitating the replacement of the dilator and further catheter manipulations to straighten out the delivery sheath. With the stent delivery catheter as part of the sheath and manoeuvring the entire complex as a unit obviates the development of the sheath kinks. This greatly eases stent placement in small infants and in stenoses with difficult access. As the sheath does not require flushing this is especially advantageous for cases needing stent placement in the systemic circulation.

In conclusion, this case shows that persistent obstruction of the pulmonary venous outlet can be successfully relieved with the use of a percutaneous intravascular stent and further expands the paediatric applications of this new therapeutic method. The long term patency of such a device within the pulmonary venous system remains unknown, although large diameter stents in high flow venous channels have previously shown optimal patency rates. ${ }^{6}$ The modifications of the delivery technique that we described have facilitated the technical aspects of stent placement.

1 O'Laughlin MP, Slack MC, Grifka RG, Perry SB, Lock $\mathrm{JE}$, Mullins CE. Implantation and intermediate term follow-up of stents in congenital heart disease. Circulation 1993;88:605-14.

2 Redington AN, Hayes AM, Ho SY. Transcatheter stent implantation to treat aortic coarctation in infancy. $\mathrm{Br}$ Heart $\mathcal{f} 1993 ; 69: 80-2$.

3 Stark J. Reoperations after Mustard and Senning operations. In: Stark J, Pacifico A, eds. Reoperations in cardiac tions. In: Stark J, Pacifico A, eds. Reoperations in

4 Coulson JD, Jennings RB Jr, Johnson DH. Pulmonary venous atrial obstruction after the Senning procedure: relief by catheter balloon dilatation. Br Heart $\mathcal{F} 1990$; 64:160-2.

5 Hosking MCK, Alsheri M, Murdison KA, Teixeira OHP Duncan WJ. Transcatheter management of pulmonary venous pathway obstruction with atrial baffle lea following Mustard and Senning repair. Cathet Cardiovas Diagn 1993;30:76-82.

6 Chaitelain P, Meier B, Friedli B. Stenting of superior ven cava and inferior vena cava for symptomatic narrowing after repeated atrial surgery for D-transposition of the great vessels. Br Heart $f$ 1991;66:466-8. 